\title{
ANALISIS DE LA COMUNIDAD DE MALEZAS EN TRIGO CULTIVADO SOBRE DISTINTOS ANTECESORES ${ }^{1}$
}

\author{
EDUARDO REQUESENS ${ }^{2}$, CESAR MORAN ${ }^{3}$, ALBERTO PASQUALINI ${ }^{3}$ y MARISA NUCIARI ${ }^{4}$
}

\section{RESUMO}

Comparou-se a composição e frequiência relativa das espécies e dos índices de diversidade e dominância da comunidade infestante de três áreas de trigo que foram cultivadas com trigo, milho e batata anteriormente. $\mathrm{O}$ estudo foi realizado na região de Mar del Plata, Província de Buenos Aires Argentina. Em cada área, realizou-se 40 amostragens de $1 \mathrm{~m}^{2}$, dispostos de forma regular no campo, antes da aplicação dos herbicidas. Foram observadas pequenas diferenças entre as três comunidades florísticas levantadas, porém importantes mudanças foram verificadas na freqüência relativa de algumas espécies. Stellaria media foi a espécie mais freqüente, quando a cultura foi precedida de trigo e milho e sendo apenas superada por Solanum tuberosum quando a cultura do trigo teve como antecessor a cultura da batata. Algumas espécies como Chenopodium album, Zea maíz, Solanum tuberosum, Polygonum aviculares, Ammi viznaga, Veronica persica e Taraxacum officinalis incrementaram suas frequiências relativas nas áreas de trigo precedidas de milho e batata. Por

outro lado, a frequiência relativa de outras espécies como Apium leptophyllum, Polygonum aviculares e Matricaria chamomilla decresceram nos mesmos locais. As mudanças específicas nas frequiências relativas não afetaram os

parâmetros estruturais das comunidades infestantes, os quais mostraram valores similares de alta diversidade específica e baixa dominância.

Palavras chave: Sequiência de cultivos, freqüências relativas, diversidade específica, Argentina.

\section{A B S T R A C T}

\section{Analysis of weed community in wheat crop grown on different precedent crops}

Relative frequency of species, diversity and dominance indexes of the weed community in three wheat crops grown in fields of 40 ha where wheat, corn or potato were grown in the previous season, were analyzed. The study was performed in Mar del Plata, Province of Buenos Aires (Argentina). The ocurring species were registred in each of forthy $1 \mathrm{~m} 2$ samples distributed in a regular arrangement at each field. This was done previous to application of herbicides. Small differences between communities in floristic composition and richness were observed. But, important changes in the relative frequency of some species were observed. Stellaria media was the most frequent specie in wheat-wheat and cornwheat sequences, and was only exceeded by Solanum tuberosum in potato-wheat sequence. The relative frequency of some species such as Chenopodium album, Zea maiz, Solanum tuberosum, Polygonum aviculare, Ammi viznaga, Veronica persica and Taraxacum officinalis was increased in wheat crop cultivated on corn or

1 Recebido para publicação em 14/07/95 e na forma revisada em 02/06/96.

2 Professor del Departamento de Ecologia de la Facultad de Agronomia (UNCPBA). CC 178 (7300) Azul, Provincia de Buenos Aires (Argentina).

3 Estudiantes de la Facultad de Ciencias Agrarias (UNMP).

4 Professora de Botánica de la Facultad de Ciencias Agrarias (UNMP). CC 276 (7620) Balcarce, Provincia de Buenos Aires (Argentina) 
potato. On the other hand, the relative frequences of other species such as Apium leptophyllum, Polygonum convolvulus and Matricaria chamomilla decreased in the same sites. The specific changes in the relative frequences did not affect the structural indexes of weed communities,

\section{INTRODUCCION}

Gran parte de la infestación de malezas en sistemas cultivados se origina en el ban co de se millas del suelo. Dado que el mismo está conformado por grupos de especies con diferentes patrones temporales de emergencia (Roberts y Feast, 1970), la expresión de la comunidad de malezas en términos florísticos y estructurales, está condicionada primariamente por la estacionalidad climática (Post, 1988; Requesens y Madanes, 1989; Requerens et al., 1992). Así, la época de siembra de los cultivos es uno de los princi pales factores que determina la flora acompañante. No obstante, otros factores como las prácticas culturales anteriores a la implantación de cultivo pueden in troducir cambios importantes dentro de un a misma estación (Pollard y Cussans, 1981; Fr ou dWilliams et al., 1983). La secuencia de cultivos integra el conjunto de dichas prácticas y sus efectos sobre la comunidad de malezas han sido evaluados para diferentes si stemas agrícolas (Johnson y Coble, 1986; Hill et al., 1989; Ball y Miller, 1990; Hume et al., 1991).

El objetivo de este trabajo es analizar aspectos cual itativos y c .antitativos de la comunidad de malezas de Ligo cultivado sobre distintos antecesores en el área mixta papera del sudeste de la Provincia de Buenos Aires. En la misma, el cultivo de trigo se realiza frecuentemente sobre antecesores estivales, practicándose ocasionalmente la secue ncia trigo-trigo.

\section{MATERIALES Y METODOS}

El estudio se realzó en un campo ubicado which showed a similar and high diversity inde $x$ and low dominance.

key words: Weed flora, crop sequences, relative frequency of species, species diversity, Argentina.

en cercanías de la localidad de Mar del Plata (Provincia de Buenos Aires), correspondiente al área mixta papera de la Re gión Pampeana Húmeda Sur (D arwich, 1991). en el mismo, fueron seleccionados tres lotes de 40 hectáreas cada uno, edáficamente similares y sembrados con trigo a mediados del mes de Julio. Anteriormente, los lotes habían sido cultivados con trigo, maíz y papa, respectivamente. El primero, representativo de cultivos inverno-primaverales y suje to a control químico de male zas con herbicidas hormonales, y los dos restantes, representativos de cultivos primavero-estivales y sujetos a controles mecánicos de male zas. En cada lote y siguiendo un diseño de muestreo regular, se analizáron 40 muestras de $1 \mathrm{~m}^{2}$ en las que se registraron las es pecies presentes previo a la aplicación de herbicidas.

A partir del muestreo realizado se de te rminó, para cada es pecie, la frecue ncia absoluta (nú mero de muestras en que fue detectada) y Ia frecuencia relativa (porcentaje de muestras en que fue detectada). Las especies fueron caracterizadas además por la forma de vida según Raun kiaer y por el ciclo estacional de crecimiento, clasificándoselas como invernoprimaverales o primavero-estivales en base a Alonso (1984).

Las comunidades fueron comparadas a partir de la composición y frecuencia relativa de las especies. Para esto último, las especies correspondientes al lote de trigo sobre trigo fueron ordenadas de mayor a menor y la secuencia resultante fue mantenida para Ias dos situaciones restantes a fin de analizar las variaciones específicas. Finalmente, y utilizando las frecuencias absolutas como valores de imp ortancia, se calcular on los índices de 
diversidad de Shannon-Weaver y de dominancia de Simpson (Mueller-Dubois y Ellenberg, 1974).

\section{RESULTADOS Y DISCUSION}

Un total de 23 especies, en su mayoría terófitas inverno-primaverales, fueron detectadas en el conjunto de los tres lotes (Tabla 1). La totalidad de Ias mismas fueron registradas en el lote de trigo sobre maíz. La mayoría de ellas fue registrada en los dos lotes restantes, con excepcíon de Zea maiz en trigo sobre trigo y Zea maiz junto con Apium leptophyllum en trigo sobre papa.

TABLA 1. Listado, forma de vida y ciclo estacional de crecimiento de las especies registradas en lotes de trigo cultivado sobre distintos antecesores.

\begin{tabular}{llcc}
\hline & \multicolumn{1}{c}{ ESPECIE } & FORMA DE VIDA & CICLO DE CRESCIMENTO \\
\hline 01 & Ammi majus & terófita & inverno-primaveral \\
02 & Ammi viznaga & terófita & inverno-primaveral \\
03 & Anagallis arvensis & terófita & inverno-primaveral \\
04 & Apium leptophyllum & terófita & inverno-primaveral \\
05 & Brassica campestris & terófita & inverno-primaveral \\
06 & Capsella bursa-pastoris & terófita & inverno-primaveral \\
07 & Carduus acanthoides & terófita & inverno-primaveral \\
08 & Chenopodium album & terófita & primavero-estival \\
09 & Matricaria chamomilla & terófita & inverno-primaveral \\
10 & Plantago lanceolata & hemicriptófita & inverno-primaveral \\
11 & Polygonum aviculare & terófita & inverno-primaveral \\
12 & Polygonum convolvulus & terófita & inverno-primaveral \\
13 & Portulaca oleracea & terófita & primavero-estival \\
14 & Raphanus sativus & terófita & inverno-primaveral \\
15 & Rapistrum rugosum & terófita & inverno-primaveral \\
16 & Rumex crispus & hemicriptófita & inverno-primaveral \\
17 & Solanum tuberosum & geófita & primavero-estival \\
18 & Stellaria media & terófita & inverno-primaveral \\
19 & Taraxacum officinalis & hemicriptófita & inverno-primaveral \\
20 & Trifolium repens & hemicriptófita & inverno-primaveral \\
21 & Veronica persica & terófita & inverno-primaveral \\
22 & Viola arvensis & terófita & inverno-primaveral \\
23 & Zea mays & terófita & primavero-estival \\
\hline & & & \\
\hline
\end{tabular}

Diferencias más pronunciadas fueron observadas en la frecuencia relativa de las especies, fundamentalmente entre el antecesor trigo y ambos antecesores estivales (figuras 1, 2 y 3). Stellaria media resultó ser la especie más frecuente con antecesor trigo y con antecesor maíz, siendo superada por Solanum tuberosum cuando el antecesor es papa. Apium leptophyllum y Polygonum convolvulus, de alta frecuencia con antecesor trigo, presentó una baja o nula presencia con antecesores estivales. Matricaria chamomilla redujo su frecuencia de casi un 50\% en trigo sobre trigo a cerca del $15 \%$ en trigo sobre maíz o papa. Otras especies, en cambio, incrementaron su frecuencia relativa con estos últimos antecesores. Tales los casos de Polygonum aviculares, Chenopodium album, Solanum tuberosum y Zea maiz en trigo sobre maíz y de Ammi viznaga, Solanum tuberosum, Veronica persica y Taraxacum officinalis en trigo sobre papa. 


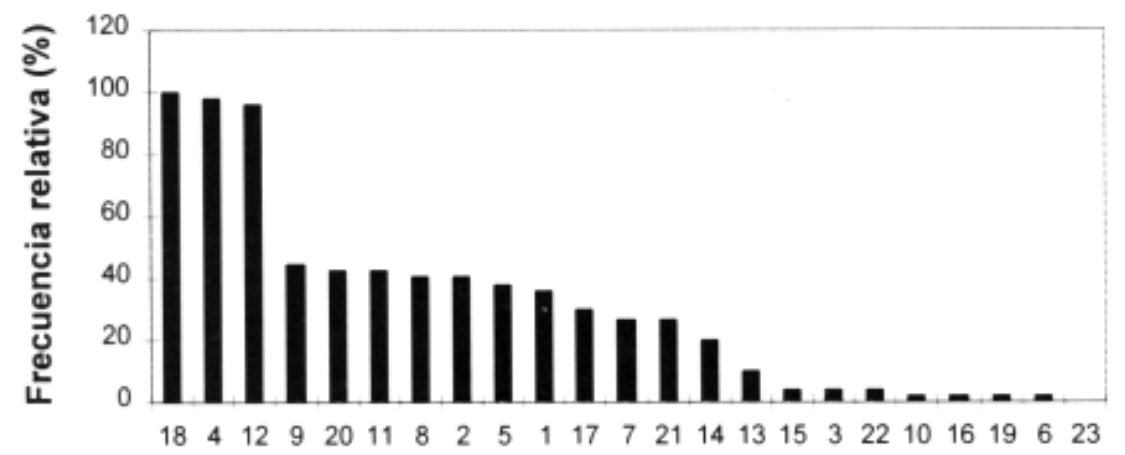

Especies

FIGURA 1. Frecue ncia relativa de Ias especies en trigo sobre trigo. Refere ncia de las especies en Tabla 1.

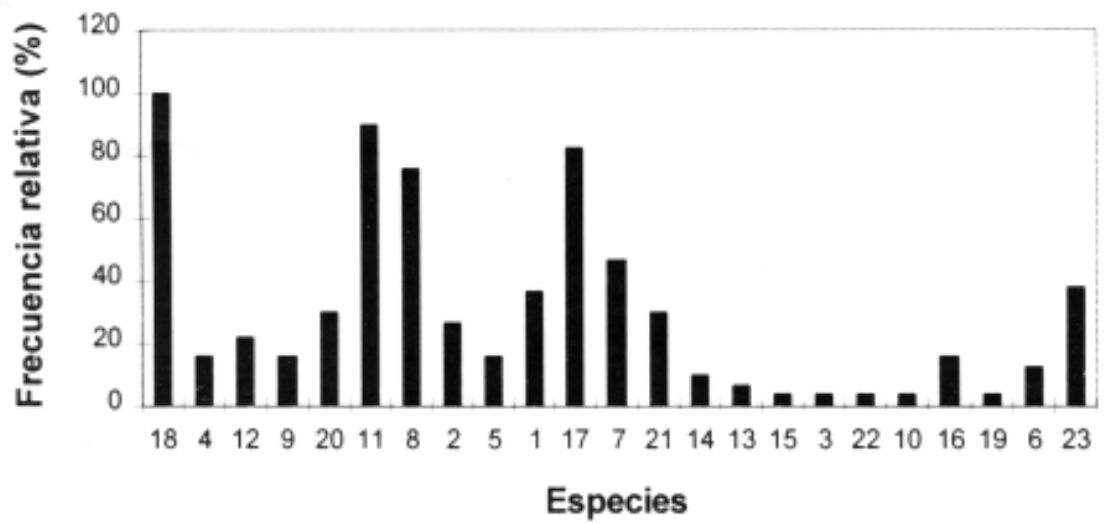

FIGUR A 2. Fre cue ncia relativa de las especies en trigo sobre maiz. Referencia de Ias especies en Tabla 1.

Estas variaciones en la frecuencia relativa de las especies pueden ser explicadas por el hecho de que las prácticas culturales asociadas a distintos antecesores pueden afectar diferencialmente la dinámica poblacional de Ias malezas (Ball, 1992). En este trabajo, la realización de cultivos estivales antes de trigo resultó ser más efectivo que el control químico en el cultivo previo de trigo para reducir la frecuencia de ciertas especies como Apium leptophyllum, Polygonum convolvulus y Matricaria chamomilla. En cambio, favorecieron a especies primavero- estivales como Zea maiz, Solanum tuberosum y Chenopodium album, y a especies invernoprimaverales como Polygonum aviculare, Ammi viznaga, Veronica persica y Taraxacum officinalis, sobre las cuales, el control con herbicidas hormonales es poco efectivo. Además, debe tenerse en cuenta que cada especie tiene requerimientos específicos para la germinación (Baskin y Baskin, 1988) y, de este modo, los cambios observados podrían estar también asociados a diferencias microclimáticas producto de diferentes historias de uso previo (Post, 1986). 


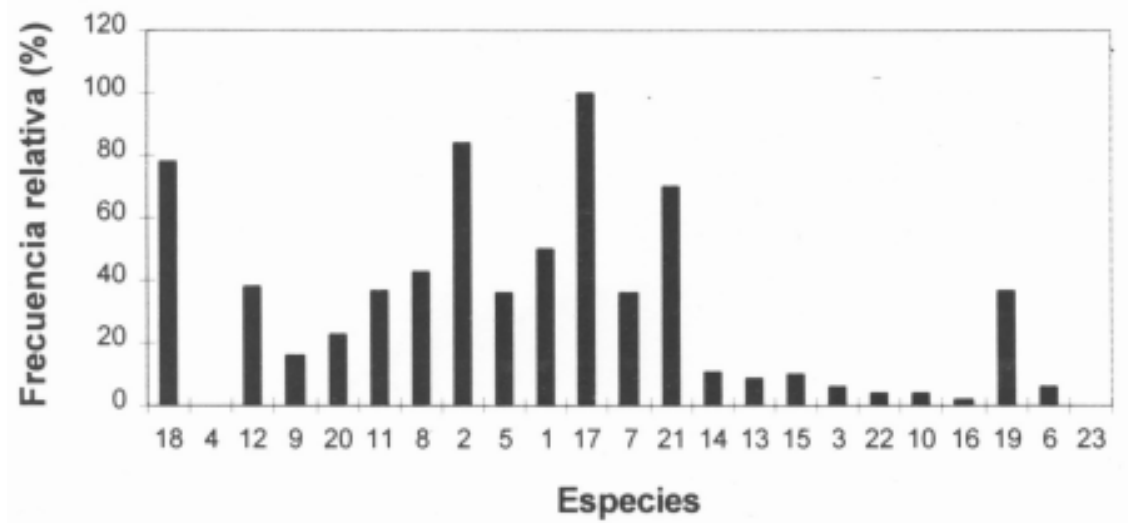

FIGURA 3. Frecuencia relativa de las especies en trigo sobre papa. Referencia de las especies en Tabla 1.

Los cambios específicos observados en las frecuencias relativas no afectaron los parámetros estructurales de la comunidad de malezas. Tanto el índice de diversidad como el de dominancia mostraron escasas diferencias entre sítios (Tabla
2). Al mis mo tiempo, los relativamente altos valores de diversidad y bajos valores de dominancia observados ponen de manifiesto un al to grado de complejidad estructural de las comunidades estudiadas.

TABLA 2. Indice de diversidad específica (Shannon-Weaver) y indice de dominancia (Simpson) de la comunidad de malezas en lotes de trigo con diferentes antecesores.

\begin{tabular}{lcc}
\hline \multicolumn{1}{c}{ SITIOS } & DIVERSIDAD & DOMINANCIA \\
\hline Trigo sobre trigo & 2.6994 & 0.0828 \\
Trigo sobre maíz & 2.7362 & 0.0827 \\
Trigo sobre papa & 2.7235 & 0.0779 \\
\hline
\end{tabular}

\section{BIBLIOGRAFIA}

ALONSO, S. Período de emergencia de Ias principales malezas del sudeste bonaerense. INTA - Estación Experim e n t a l Agropecuaria Balcarce, informe para extensión, producción veg etal, v.2, n.14, p.4, 1984.

BALL, D.A. Weed seedbank response to tillage, herbicides, and crop rotation sequence. Weed Sci., v.40, p.654-659, 1992.

BALL, D. A., MILLER, S. D. Weed seed population response to tillage, and herbicide use in three irrigated cropping sequences. Weed Sci., v.38, p.511-517, 1990.

BASKIN, C.C., BASKIN, J.M. Germination ecophysiology of herbaceous plant species in a temperate region. Amer. J. Bot., v.75, p.286-305, 1988.

DAR WICH, N. Estado actual y manejo de los recursos naturales en la Región Pampeana Hú me da Sur. En: In stitut o Nacional de Tecn ol ogía Ag ropecuaria, Ed., Juicio a nuestra agricultura, Impresiones Sud 
América, Buenos Aires, 1991. p.53-62

FROUD-WILLIAMS, R.J., DRENNAN, D.S.H., CHANCELLOR, R.J. Influence of cultivation regime on weed floras of arable cropping systems. J. Appl. Ecol., v.20, p.187-197, 1993.

HILL, N.M., PATRIQUIN, D.G., VANDER KLOET, S.P. Weed seed bank and vegetation at the beginning and end of the first cycle of a 4-course crop rotation with minimal weed control. J. Appl. Ecol., v.26, p.233-246, 1989.

HUME, L., TESSIER, S., DYCK, F. Tillage and rotation influences on weed community composition in wheat southwestern Saskatchewan. Can. J. Plant Sci., v.71, p.783-789, 1991.

JOHNSON, C., COBLE, H. Crop rotation and herbicide effects on the population dynamics of two annual grasses. Weed Sci., v.34, p.452-456, 1986.

MUELLER-DOMBOIS, D., ELLEMBERG, H. Aims and methods of vegetation ecology. John Wiley \& Sons, New York, 1974.
POLLARD, F., CUSSANS, G.W. The influence of tillage on the weed flora in a succession of winter cereal crops on a sandy loam soil. Weed Res., v.21, p.185-190, 1981.

POST, B.J. Factors of influence on the development of an arable weed vegetation. Proceedings of the EWRS Symposium, Economic Weed Control, StuttgarHohenheim, p.317-325, 1986.

POST, B.J. Multivariate analysis in weed science. Weed Res., v.28, p.425-430, 1986.

RO BERT S, H. A., FE AS T, P.M. Se as on al distribution of emergence in some annual weeds. Expl. Hort., v.21, p36-41, 1970.

REQUESENS, E., MADANES, N. Organización de comunidades estacionales de malezas en el sudeste de la Provincia de Buenos Aires. Ecología Austral, v.2, p.101-108, 1992.

REQUESENS, E., MADANES, N., MONTES, L. Análisis de la composición florística y de la dinámica de dos comunidades estacionales de malezas. Revista de la Facultad de Agronomía (La Plata), v.65, p.53-60, 1989. 